\title{
Assessing clinical competency in medical senior house officers: how and why should we do it?
}

\section{S J Carr}

Postgrad Med J 2004;80:63-66. doi: 10.1136/pmi.2003.011718

Most consultants are involved in the training and assessment of several grades of doctors in training especially senior house officers (SHOs) and specialist registrars. In the medical and other specialties there is an increasing trend towards assessing junior doctors' competency using the record of in-training assessment process for specialist registrars and using the Royal College of Physicians folder to record competences of medical SHOs.It is necessary to consider why there is a need to assess competency, how it may be done practically, and the advantages and disadvantages of this system of assessment. There are considerable hurdles to the implementation of this system in the medical specialties within today's NHS and the organisation may need to undergo fairly radical change to facilitate this system.

Correspondence to:

Dr S J Carr, Department of Nephrology, University Hospitals of Leicester NHS Trust, Leicester General Hospital, Gwendolen Road, Leicester LE5 4PW, UK; sue.carr@uhl-tr.nhs.uk

Submitted 23 June 2003 Accepted 23 July 2003

\section{WHY ARE WE MOVING TOWARDS COMPETENCY BASED MEDICAL TRAINING?}

Patients expect doctors to be competent to diagnose, plan management, carry out practical procedures, and they expect them to behave in a reasonable way demonstrating a caring and humanistic attitude while doing so.

Unfortunately, following a series of high profile medical incidents the government, the media, and the public have become concerned about the quality of clinical care and have focused their attentions on the system by which we demonstrate clinical competence. It is generally recognised that it is no longer sufficient for doctors to consider themselves competent, but that there is a need for professionals to demonstrate they are clinically competent to perform certain roles. In addition, it is now a requirement of the Clinical Negligence Scheme for Trusts that an assessment of the competencies of doctors in training is undertaken in a more formalised fashion. Failure to meet this standard has serious financial implications for NHS trusts as it is directly related to the insurance a trust can obtain against medical litigation claims.

It is therefore increasingly important to incorporate this principle into doctors' early clinical training and for the medical profession to begin to assess and document competencies in a more formalised fashion.

This increasing emphasis towards a competency based training system is not unique to the
$\mathrm{UK}$; in the United States and in other countries around the world there is a pressure to increase accountability and to formalise the maintenance of standards as well as setting standards for entry into practice. ${ }^{1}$ The American Council for Graduate Medical Education is planning for all graduate medical education to be competency based. In the UK competency based training is currently centre stage both in government policy, the media, and a number of Royal Colleges have produced competency based curricula for senior house officers (SHOs)-for example, the Royal College of Anaesthetists.

\section{THEORETICAL BASIS FOR ASSESSING COMPETENCE}

Over recent years educational theorists have published several models to describe the development of knowledge and the educational processes involved in achieving competence. Some authors have applied these theoretical constructs to the development of clinical competence.

Over 20 years ago Rasmussen proposed a scheme to define learning in situations where rapid decisions have to be made often without all the necessary information that would be desirable $^{2} 3$ - a situation often encountered in medicine. He suggested that the first stage in practical learning is the acquisition of skills and that one can be competent in these skills before the full knowledge relating to the skill has been acquired. The skills can then be applied by students using a series of rules. He theorises that with time the practical experience increases and is augmented with knowledge. Ideally the learner would eventually move to the highest level of "knowledge based practice". Rasmussen's hypotheses are discussed in current literature, ${ }^{23}$ and this theory is quoted by Long as the basis for the more recent introduction of competency based training in medicine. ${ }^{4}$ Rasmussen's construct is more applicable to the procedural specialties than to the work of physicians and others where the use of practical procedures is quite small. In my opinion this construct moves away from encouraging development of global professional skills in a doctor to describing a "vocational training" and personally I would see this approach as detrimental to medical education.

Abbreviations: $M C Q$, multiple choice question; miniCEX, mini-clinical evaluation exercise; OSCE, objective structured clinical examination; $\mathrm{SHO}$, senior house officer 
Other models have been proposed and applied to learning in clinical medicine. Dreyfus and Dreyfus developed a five stage model initially to describe the development of knowledge and skills of a pilot. ${ }^{5}$ In a recent paper they attempt to expand this model to cover the acquisition of clinical skills in medicine $^{6}$ (table 1) and to encompass some aspects of "performance". A more useful model was proposed by Miller in his well known triangle (fig 1). ${ }^{7}$ He identifies four stages of development "knows, knows how, shows how, and does" as the cognitive and behavioural steps an individual progresses through from acquiring knowledge to performing a task in practice. Miller's triangle assumes that competence predicts performance which may not always be the case. In clinical practice many other factors may influence clinical performance including time availability, tiredness, mood of the doctor and patient, etc. Knowing and showing does not mean that a doctor will perform in a certain way in real practice. To address these concerns Rethans and colleagues proposed a modification to Miller's triangle: "The Cambridge model". ${ }^{8}$ They aimed to broaden Miller's triangle to define the factors influencing "performance" in addition to competence. They ascertain that competence is what a doctor demonstrates in a test situation but that performance is what a doctor demonstrates in real clinical practice. Performance builds upon competence but also encompasses other influences on one's eventual performance, including system related influences (government programmes and initiatives, patient's expectation, guidelines, etc) and individual related influences (physical and mental health, relationship with others including peers, other professions, and family). They produced a modified triangle as shown in fig 2. This model acknowledged that in addition to assessing knowledge and practical skill we need to assess the global competence or performance of doctors in training in as realistic a way as possible.

\section{WHAT IS CLINICAL COMPETENCE AND HOW SHOULD CLINICAL COMPETENCE OF DOCTORS IN TRAINING BE ASSESSED?}

Defining what clinical competence actually is and then devising methods to reliably and feasibly assess it has proved difficult.

Table 1 Dreyfus stages applied to clinical medicine ${ }^{5}$

\section{Dreyfus stages \\ Stage 1-novice (medical}

student)

Stage 2-advanced beginner (house officer or $\mathrm{SHO}$ ) appointed consultant)

physician)
Learns basics of history taking and examination

Learns to apply skills in selected clinical situations which become increasingly dependent on the context of the situations - that is, hospital admissions, rounds, etc, which enables learning through experience

Stage 3-competent (registrar)

Learns to plan the approach to each patient's situations in a supervised fashion. Learns consequences of actions and pattern recognition

Develops routines to streamline patient care. Manages multiple stimuli in a thoughtful way which is intellectually and emotionally absorbing. Integrates mastered skills with personal style

Recognises patterns, has intuition for the work, attuned to distortions in patterns and to slow down when things don't fit the expected pattern

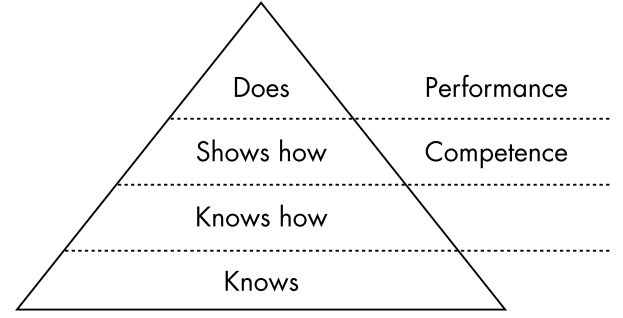

Figure 1 Miller's triangle (Miller ${ }^{7}$; published with permission).

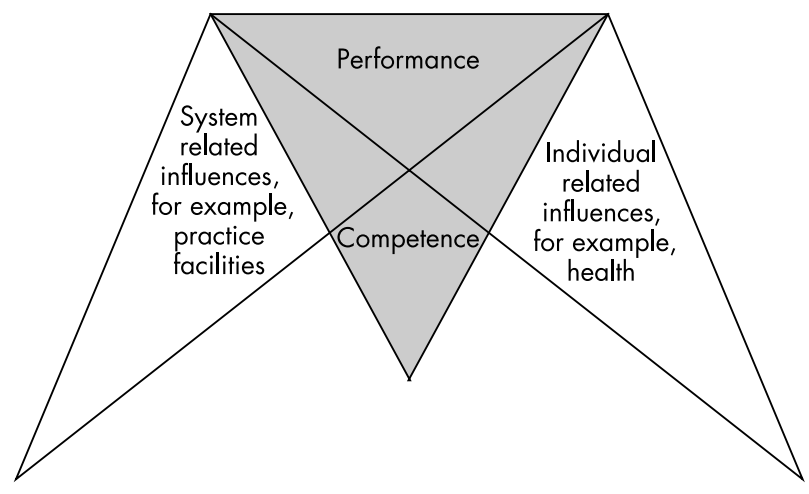

Figure 2 The Cambridge model for delineating performance and competence (Rethans et $a^{\beta}$; published with permission).

In the literature there are many definitions of clinical competence, some simple and some more complex: Southgate defined competence in a doctor as being "composed of cognitive, interpersonal skills, moral and personality attributes. It is in part the ability, in part the will, to consistently select and perform relevant clinical tasks in the context of the social environment in order to resolve health problems of individuals in an efficient, effective economic and humane manner". ${ }^{1}$ It is important to emphasise that competence is always context based. Epstein and Hundert defined competence in a more complex way as "the habitual and judicious use of communication, knowledge, technical skills, clinical reasoning, emotions, values, and reflection in daily practice for the benefit of the individual and community being served". ' Others would argue that this is "performance" rather than purely "competence".

Competence builds on a foundation of basic clinical skills, scientific knowledge, and moral development. It has several different domains':

- Cognitive function: acquiring and using knowledge to solve problems.

- Integrative function: using biomedical and psychosocial data in clinical reasoning.

- Relational function: communicating effectively to patients, carers, and colleagues.

- Affective and moral function: willingness, patience, emotional awareness to use these skills judiciously and humanely.

Therefore, having defined competence we need to consider the methods of assessment available to us and suitable to apply to SHOs.

The central features of assessing clinical competence are to:

- Establish the range of knowledge, skill, and understanding that an individual should have achieved at a certain stage of their career. 
- Design tasks that appropriately and accurately sample and estimate level of competence.

- Establish cut off points to separate the competent from the not yet or barely competent.

Assessment of knowledge as defined in stages 1 and 2 of Miller's pyramid (fig l) is generally performed using by traditional assessment tools including written and oral tests. ${ }^{10}$ In medical, surgical, and the majority of secondary care specialties this is assessed by the written examinations of the Royal Colleges involving multiple choice questions (MCQs), best of five questions, extended matching, and short answer questions. An assessment of clinical reasoning is made in the Royal College of Physicians examination by the use of "grey cases", which are structured complex cases. It is important that these assessment methods evolve to become better tools to assess physicians' competence as well as core knowledge. One study described the development of open ended MCQs (UnQ) and reported that this technique differentiated experienced and junior physicians. ${ }^{11}$

However, as previously discussed, knowing and knowing how, do not always extrapolate to application of knowledge in the workplace or clinical performance. Assessing levels 3 and 4 of Miller's triangle is more challenging (figs 1 and 2). At the present time level 3 in medical specialties is currently assessed by practical clinical examinations including objective structured clinical examinations (OSCEs). ${ }^{12}{ }^{13}$ Several other methods have been devised and validated including the mini-clinical evaluation exercise (mini-CEX) ${ }^{14}$ and/or performance evaluation guides. ${ }^{15}$ This type of assessment can be used in a formative or a summative fashion provided the content of the tasks is tailored to the purpose of the examination. The validity of an OSCE and other assessment tools depends upon the number of stations and skills evaluated to give an overall view of the trainees' clinical skill level. When designing an OSCE or other clinical assessment tool it is extremely important to test the reliability, validity, and feasibility of the tool especially if the examination planned is a high stakes examination and will be conducted at different sites.

Sloan et al found the OSCE to be a useful tool in assessing house staff and in identifying curriculum deficiencies in a training programme. ${ }^{16}$ In the future, in view of the large number of assessments to be undertaken across many different sites, information technology may be utilised. Novack et al described the development of a webOSCE system for assessing clinical skills via teleconference. ${ }^{17}$ Using the internet and videoconferencing facilities they staged the same clinical examination at several centres simultaneously. They reported technical difficulties with the system and students found lack of direct contact with the patients difficult. There are other papers describing computer systems in the evaluation of clinical competence, ${ }^{18}$ and in the future such technologies may impact upon assessment systems in clinical education. Other computer based methods of clinical assessment are described in the literature. One method is the "Primium ${ }^{\odot}$ Simulations" in which candidates navigate through a clinical scenario on the computer cued only by evolving facts. Candidates are scored on their choices of testing and therapy and the assessor attempts to determine the clinical reasoning ability of candidates. ${ }^{19}$

The most difficult facet of clinical competence to examine is level 4 in Miller's triangle- "does" or performance. However, even if we have tools to adequately assess performance in a test environment this does not necessarily assess what physicians really do in practice. It is important to directly observe trainee physicians to ensure effective assessment of clinical skills. This type of assessment can be time consuming and costly. The Royal College of General
Practitioners employs a system of observing a collection of videotaped consultations submitted by the trainee to demonstrate a range of clinical skills. Several authors advocate the use of standardised patients in these assessments, ${ }^{20}{ }^{21}$ but great care must be given to the training of patients, construction of valid cases, and scoring scales. ${ }^{19}$ In the United States candidates undergo 10 simulated patient observations for the clinical skills assessment required for Educational Commission for Foreign Medical Graduates certification. ${ }^{21}$ The American Board of Internal Medicine uses a different system to evaluate the clinical competence of residents in the mini-CEX which scores the clinical skills that residents demonstrate in patients encounters. The assessments use real clinical situations and are scored by supervising physicians or chief residents. As in all clinical examinations the ratings could be influenced by a particular examiner or the patients encountered therefore the residents have multiple 15-20 minute observations. Boulet et al recently reported correlation between scores using the two systems. ${ }^{21}$

The assessment of attitude poses a difficult area in which to assess competence. In addition to observed clinical interactions other methods can be employed to assess a doctor's attitude. The use of structured or open questionnaires completed by members of the healthcare team has recently been used to inform 360 degree appraisal for medical staff. This technique is being piloted in some specialties by the Royal College of Physicians to assess specialist registrars. Attitude may also be assessed to some degree from personal logs and portfolios and may be useful tools.

To make the best evaluation of global clinical competence it is probably necessary to use a combination of different assessment methods described above. The Royal College of Anaesthetists have adopted such an approach in the evaluation of the new competency based curriculum for SHOs in anaesthesia. ${ }^{22}$ SHOs' $^{\prime}$ knowledge is assessed by a series of written examinations (FFRCA); clinical skills, attitudes, and behaviours are assessed in a workplace assessments performed by supervising consultants.

In my view, in the future, in addition to the examinations of the Royal College of Physicians, we should look to assessing performance of SHOs in medicine in the workplace using some of the tools described above. A combination of a series of mini-CEX style assessments, assessments of practical skills, and 360 degree appraisal would provide useful assessment tools. This is increasingly important in SHO training as in view of the reduced hours of work and shift working patterns the competence of an individual cannot be guaranteed by one supervising consultant and a robust system for assessing competence needs to be implemented in the future.

\section{HOW WILL COMPETENCY BASED TRAINING CHANGE EDUCATION FOR DOCTORS IN TRAINING?}

It is frequently stated that "If you want to change student learning then change the assessment process". There is much evidence available in the literature to support this statement. ${ }^{15}$

Therefore, if we move to adopt a competency based education system for all postgraduate trainees this redefines the outcome of training and there will be a need to redesign educational programmes. Currently, postgraduate training programmes are based on a system whereby the training experience is defined by the exposure to content over a certain period of time-for example, six months of cardiology. A change to a competency based assessment system would logically lead away from a fixed time period in training to one in which the trainee remains in training until they 
Table 2 Comparison of key points of a time based versus a competency based approach to $\mathrm{SHO}$ training in medical specialties

\begin{tabular}{ll}
\hline Years in training approach & Competency based training \\
\hline $\begin{array}{l}\text { Specified number of years } \\
\text { assumed to acquire specific skills } \\
\text { of specialty }\end{array}$ & $\begin{array}{l}\text { Time taken to acquire the required } \\
\text { knowledge, skills, attitudes } \\
\text { irrespective of time period }\end{array}$
\end{tabular}

No specific standardised measure Competency judged by programme to document competency director using a number of (satisfactory progress documented standardised requirements by educational supervisor)

MRCP examination usually completed at end of two years' training

No limits on time in training (define maximum and minimum)

Trainees may not feel competent ?Examination in specialty at end of designated time period receives is not uniform as each doctor is exposed to a variable number and type of medical specialties as they go through a two year training rotation or a series of six month posts. This problem strengthens the need for a formal training programme and a formative type assessment as training progresses. A rigorous competency based assessment system would require a considerable increase in time from programme directors and educational supervisors to plan, administer, and document assessment of competence. This factor needs to be considered for all grades of doctors in training. Manpower planning groups estimate that one consultant session per trainee will be required per week to implement competency assessment. In my own teaching hospital department this will equal 13 sessions per week at least. These factors need to be considered by NHS planners and managers as this increased workload will take consultants away from the provision of clinical service and will reduce the impact of the governments proposed increase in consultant numbers on patient care.

\section{REFERENCES}

1 Southgate L. Professional competence in medicine. Hospital Medicine 1999:60:203-5.

have been shown to be "competent" to progress to the next stage.

This type of change in postgraduate medical education in the UK would require enormous changes within today's NHS as doctors in training continue to provide a significant service contribution. The scenario where trainees were present within a specialty for unspecified period of time-that is, until they became competent, would require significant reorganisation of the current systems (table 2) and SHOs would probably need to be supernumery.

\section{CONCLUSION}

There are several advantages offered by competency based assessment. This system improves the public accountability of medicine and the standards are transparent. The assessment process when used in a formative way may identify deficiencies, problems, and gaps in training which often arise due to variable or reduced clinical exposure as a result of reduced hours of work under the European Working Time Directive (a problem which is likely to increase as hours of work decrease in forthcoming years), it also maximises use of training opportunities as trainee and trainer know what they need to learn. Another advantage of the system would be that individuals could progress at their own pace and the system could be flexible to individual training needs.

There are, however, potential disadvantages too. One fears that by reducing each clinical skill into a list of subcomponents that the connections between these tasks may not be made and the system may not adequately assess the global or metacompetencies which a doctor needs-for example, such as how someone responds in an emergency. ${ }^{23}$ In this review article, Leung criticised competency based assessment as politically motivated allowing the government to define important competencies and allocate resources based on the outcomes of performance. ${ }^{23}$ The system could also be considered rigid and impersonal and for some individuals this could be demotivating.

There are also practical difficulties with the implementation of assessment of clinical competencies for SHOs within the NHS in its current form. At present, the experience a SHO
2 Rasmussen J, Lind M. A model of human decision making in complex systems and its use for design of system control strategies. Riso National Laboratory report. Denmark: Roskilde, Riso-M-2349, 1982.

3 Rasmussen J. Skills, rules, knowledge, signals, signs and symbols and other distinctions in human performance models. IEEE Trans Systems. Man and Cybernetics 1983;123:257-66.

4 Long D Competency-based residency training: the next advance in graduate medical education? Acad Med 2000;75:1178-83.

5 Dreyfus H, Dreyfus S. Mind over machine. New York: Free Press, 1986.

6 Batalden P, Leach D, Swing S, et al. General competence and accreditation in graduate medical education. Health Affairs 2002;21:103-11.

7 Miller GE. The assessment of clinical skills/competence/performance. Acad Med 1990;65(suppl):S63-67.

8 Rethans JJ, Norcini JJ, Baron-Maldonaldo M, et al. The relationship between competence and performance: implications for assessing practice performance. Med Educ 2002;36:901-9.

9 Epstein RM, Hundert EM. Defining and assessing professional competence. JAMA 2002;287:226-34.

10 Glavin RJ, Maran NJ. Development of a scoring system for assessment of clinical competence. Br J Anaesth 2002;88(3): 1-2.

11 Veloski JJ, Rabinowitz HK, Robeson MR, et al. Patients don't present with five choices: an alternative to multiple-choice tests in assessing physicians' competence. Acad Med 1999;74:639-46.

12 Harden R, Gleeson F. Assessment of clinical competence using an objective structured clinical examination (OSCE). Med Educ 1979;13:41-54.

13 Harden R, Cairncross C. Assessment of practical skills. Studies in Higher Education 1980:5:187-96.

14 Delisa JA. Evaluation of clinical competency. Am J Phys Med Rehabil 2000;79:474-7.

15 Brown G, Bull J, Pendlebury M. What is assessment? Assessing student learning in higher education. London and New York: Routledge, 1997:7-20 (chapter 2).

16 Sloan DA, Donnelley MB, Schwartz R, et al. The objective structured clinical examination - the new gold standard for evaluating postgraduate clinical performance. Ann Surg 1995;222:735-42.

17 Novack DH, Cohen D, Peitzman SJ, et al. A pilot test of webOSCE: a system for assessing trainees' clinical skills via teleconference. Medical Teacher 2002;24:483-7.

18 Issenburg SB, McGaghie WC, Waugh RA. Computers and evaluation of clinical competence. Ann Intern Med 1999;130:244.

19 Klass D. Revaluation of clinical competency. Am J Phys Med Rehabil 2000;79:481-6. clinical skills of physicians. Best Practices and Benchmarking in Healthcare 1997;2:174-7

21 Boulet JR, McKInley DW, Norcini JJ, et al. Assessing the comparability of standardised patient and physician evaluations of clinical skills. Advances in Health Sciences Education 2002;7:85-97.

22 Patel BS, Feerick A. Will competency assessment improve the training and skills of trainee anaesthetists. Anaesthesia 2002;57:710-31.

23 Leung WC. Competency based medical training: review. BMJ 2002;325:693-5.
20 Chablain J, Dunnington G. Standardized patients: a new method to assess the 